\title{
Growth and magnetic properties of ultrathin Fe on Pd(110)
}

Cite as: Journal of Vacuum Science \& Technology A 19, 1182 (2001); https://doi.org/10.1116/1.1345912 Submitted: 09 October 2000 . Accepted: 11 December 2000 . Published Online: 13 July 2001

\author{
Beatriz Roldan Cuenya, J. Pearson, Chengtao Yu, Dongqi Li, and S. D. Bader
}

\section{ARTICLES YOU MAY BE INTERESTED IN}

Excitation wavelength independent sensitized $\mathrm{Er}^{3+}$ concentration in as-deposited and low temperature annealed $\mathrm{Si}$-rich $\mathrm{SiO}_{2}$ films

Applied Physics Letters 95, 231109 (2009); https://doi.org/10.1063/1.3272271

Enhanced thermal stability and nanoparticle-mediated surface patterning: $\mathrm{Pt} / \mathrm{TiO}_{2}(110)$ Applied Physics Letters 94, 083102 (2009); https://doi.org/10.1063/1.3083557

Formation, thermal stability, and surface composition of size-selected AuFe nanoparticles Applied Physics Letters 91, 113110 (2007); https://doi.org/10.1063/1.2784957

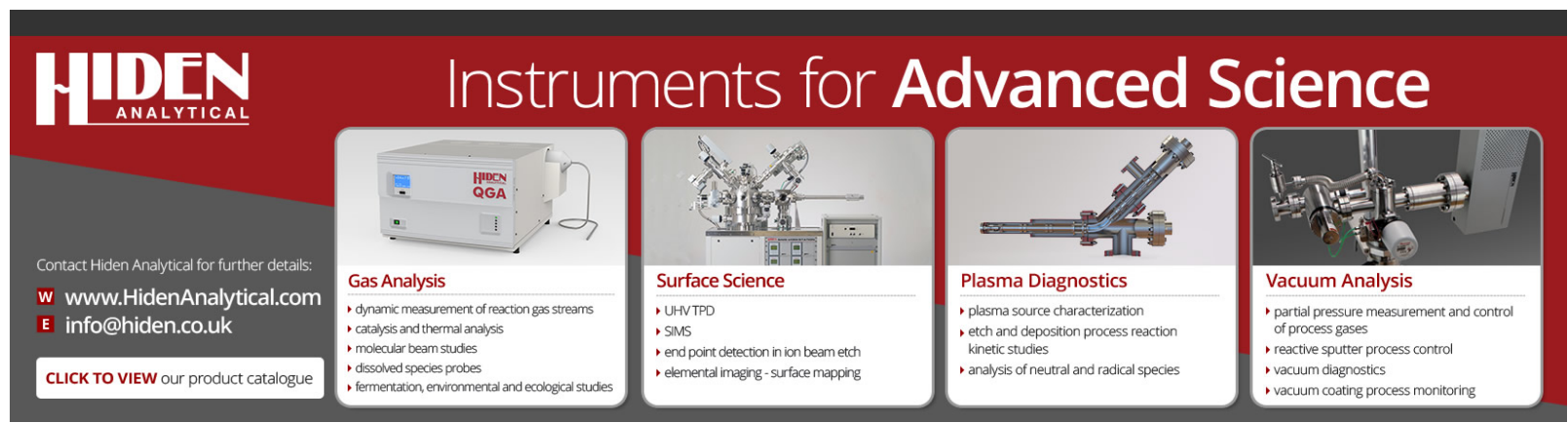




\title{
Growth and magnetic properties of ultrathin $\mathrm{Fe}$ on $\mathrm{Pd}(110)$
}

\author{
Beatriz Roldan Cuenya, ${ }^{\text {a) }}$ J. Pearson, Chengtao Yu, Dongqi Li, ${ }^{\text {b) }}$ and S. D. Bader \\ Materials Science Division, Argonne National Laboratory, Argonne, Illinois 60439
}

(Received 9 October 2000; accepted 11 December 2000)

\begin{abstract}
We have investigated the growth and magnetic properties of 0-3 ML (monolayers) Fe on stepped $\operatorname{Pd}(110)$ with reflection high-energy electron diffraction (RHEED), low-energy electron diffraction, and the surface magneto-optic Kerr effect in order to relate the morphology, structure, and magnetic properties in a low-dimensional system. The $\mathrm{Fe}$ films grown at $340 \mathrm{~K}$ are smooth and pseudomorphic up to $1.5 \mathrm{ML}$, where three-dimensional growth and lateral lattice relaxation ensues. The in-plane row spacing along the [110] direction decreases by $\sim 5 \%-6 \%$ at 3 ML. RHEED oscillations with 1 ML period are observed in the $(1,0),(2,0)$, and the center of the $(0,0)$ streak intensity. The tail of the $(0,0)$ streak at low exit angle, however, has a 0.5 ML period oscillation, which suggests step decoration growth. Submonolayer Fe films remain ferromagnetic above $\sim 0.3$ ML. The magnetic easy axis is initially perpendicular to the surface and is in-plane for Fe thickness $>1.5$ ML. Between 0.9 and 1.2 ML, there appear to be mixed magnetic phases as indicated by an increase in coercivity. (C) 2001 American Vacuum Society. [DOI: 10.1116/1.1345912]
\end{abstract}

\section{INTRODUCTION}

The field of two-dimensional (2D) magnetic ultrathin films and multilayers has advanced both in fundamental science and industrial applications thanks to new magnetic phenomena that occur at small length scales. ${ }^{1}$ Lower dimensional systems, such quasi-1D magnetic wires, however, remain less investigated due to difficulties in fabrication at the length scale $<10 \mathrm{~nm}$. Recently, self-assembly of nanowires has started to attract attention because of its potential in making regular arrays at a length scale beyond the lithographic limit. One approach is to decorate step edges of a vicinal surface by depositing metal atoms. ${ }^{2-4}$ Some promising magnetic characterizations have been reported for $\mathrm{Fe} / \mathrm{W}(110)^{5}$ and $\mathrm{Fe} / \mathrm{Cu}(111){ }^{6}$ although the wires are less than perfect.

We choose to study $\mathrm{Fe}$ on stepped $\operatorname{Pd}(110)$ for several reasons. First, the formation of smooth metallic nanowires requires not only that adatoms diffuse across terraces to stick at step edges, but also enough edge diffusion along the step edges in order to form smooth, straight chains. It is known that initial metal growth on $\operatorname{Pd}(110)$ surfaces is highly anisotropic and tends to form nanoscale wires even on flat surfaces. ${ }^{7}$ This would ensure that, on a stepped surface with the edges along the fast diffusing direction, the growth front of the wires would be straight as long as step decoration occurs. Second, Pd is known to be nearly magnetic and can have an induced magnetic moment through proximity when placed next to ferromagnetic atoms. ${ }^{8}$ This makes it more likely for the Fe wires to remain ferromagnetic. The magnetic properties of submonolayer $\mathrm{Fe}$ on $\mathrm{Pd}(110)$, to our knowledge, have not been reported before. In this work we mainly investigate the growth of $\mathrm{Fe}$ on stepped $\mathrm{Pd}(110)$ in the 0-3 monolayer (ML) region. Smooth, pseudomorphic

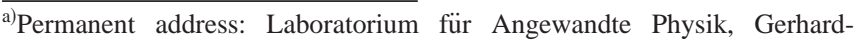
Mercator-Universität Duisburg, Duisburg, Germany.

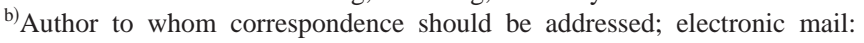
dongqi@anl.gov
}

growth is observed $<1.5 \mathrm{ML}$, where 3D growth and in-plane lattice relaxation then ensues. The peculiar reflection highenergy electron diffraction (RHEED) oscillations suggest that step decoration occurs. The system remains ferromagnetic down to $\sim 0.3 \mathrm{ML}$ and has a magnetic easy axis perpendicular to the substrate at very low coverage.

\section{EXPERIMENT}

The experiments were carried out in an ultrahigh vacuum (UHV) chamber equipped with RHEED, low-energy electron diffraction (LEED). Auger electron spectroscopy, and surface magneto-optic Kerr effect (SMOKE). The stepped $\operatorname{Pd}(110)$ was cleaned in UHV by cycles of Ar sputtering at $700 \mathrm{~K}$ and annealing at $950 \mathrm{~K}$. We also occasionally annealed the crystal in oxygen at $1 \times 10^{-7}$ Torr for several minutes to eliminate residual $\mathrm{C}$ and $\mathrm{S}$ impurities. The resultant substrate and the subsequent Fe films are free of any measurable contamination within the Auger sensitivity. Fe was evaporated with a typical rate of $0.2-0.3 \AA / \mathrm{min}$ via electronbeam bombardment of a $\mathrm{W}$ crucible filled with pure Fe. The base pressure of the chamber is $\sim 1 \times 10^{-10}$ Torr, and the pressure during deposition was $2-4 \times 10^{-10}$ Torr. Fe wedges of $0-3 \mathrm{ML}$ were grown with a slope of $\sim 0.2-0.4 \mathrm{ML} / \mathrm{mm}$ by moving the substrate behind a mask during deposition at $343 \mathrm{~K}$. The ordering of the crystal and the films was confirmed with RHEED and LEED, recorded using a chargecoupled diode camera. The images were analyzed digitally to quantify both the changes in atomic spacing along the wedges and the average terrace size of the substrate. Figure 1 shows a LEED image of the substrate. The spots are elongated due to the steps along the Pd [110]. While double spots, typical of an ordered stepped surface, are not observed, the asymmetric diffuse scattering of the spots suggests that the average terrace size is about 5-6 atomic rows, or $\sim 20 \AA$ A. Magnetic properties were studied in situ at $\sim 40 \mathrm{~K}$ by means of longitudinal and polar SMOKE. A He-Ne laser beam focused to $0.2 \mathrm{~mm}$ was used to scan along the wedge 

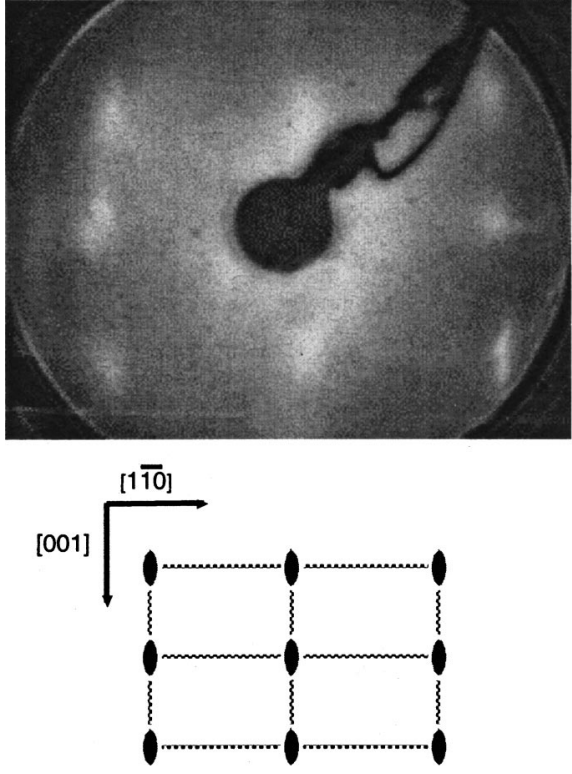

FIG. 1. LEED pattern of the Pd(110) substrate. The high symmetry directions are marked on the schematics. The elongated spots are caused by steps along the [110] as discussed in the text.

to obtain Kerr elipticity hysteresis loops for different Fe thicknesses. The height of the loops in remanence denoted $M_{R}$ is proportional to the remanent magnetization.

\section{RESULTS AND DISCUSSIONS}

Figure 2 shows typical RHEED patterns of the Pd(110) substrate and Fe films at different thicknesses, where the electron beam is along the [001] of the Pd surface. For both the clean surface and the Fe films of less than $\sim 1.5 \mathrm{ML}$, sharp streaks are observed, indicating smooth, well-ordered surfaces. Above $\sim 1.6 \mathrm{ML}$, the streaks begin to break up into spots, indicating the initiation of 3D growth. Clear RHEED patterns with similar characteristics, although increasingly
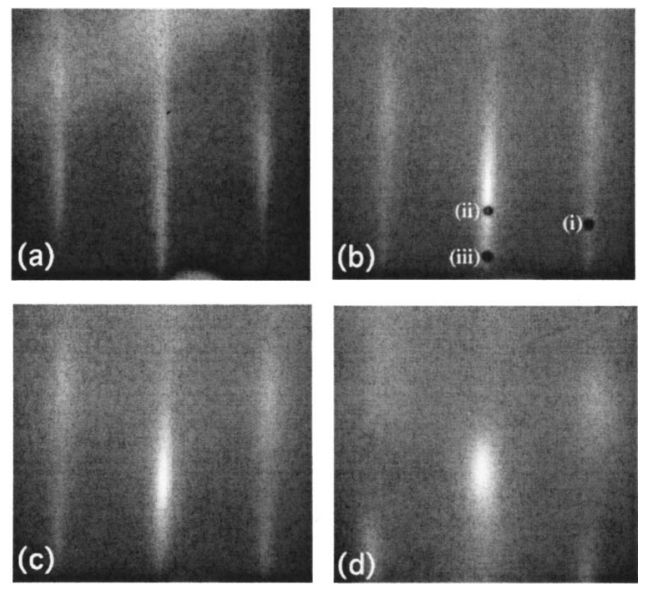

FIG. 2. RHEED images of: (a) the Pd(110) substrate, (b) $1.06 \mathrm{ML}$, (c) 1.65 $\mathrm{ML}$, and (d) $10.90 \mathrm{ML}$ of Fe on Pd(110) grown at $343 \mathrm{~K}$. Note that the streaks start to become spotty in (c), indicating 3D growth. The electron beam incidence plane is parallel to the Pd [001]. (i), (ii), and (iii) indicates the positions where RHEED oscillations are taken in Fig. 4(a).

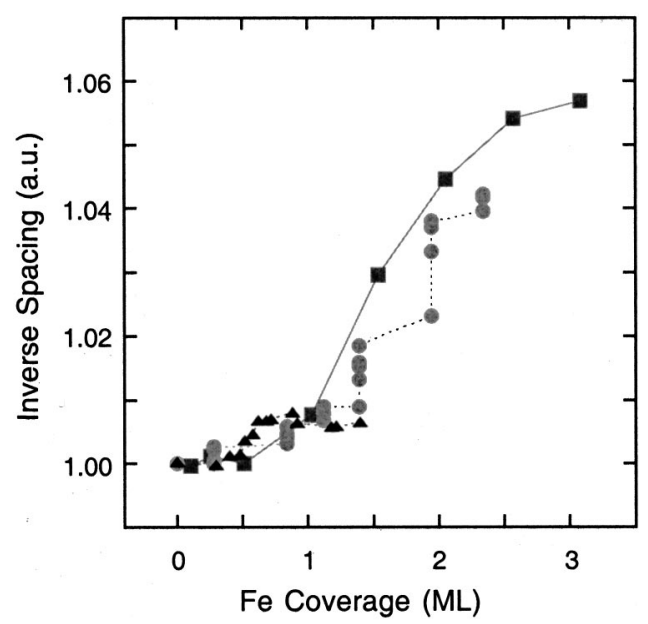

FIG. 3. Inverse lattice spacing along the Pd [110] as a function of Fe film thickness derived from a series of RHEED images taken along a Fe wedge. Different symbols indicate the results from different wedges. Significant lattice relaxation is observed after $1.5 \mathrm{ML}$ of $\mathrm{Fe}$.

spotty and diffuse, are observed at up to $11 \mathrm{ML}$ where the experiment was terminated. The distance among the streaks/ spots is proportional to the inverse lattice spacing of the surface and is plotted in Fig. 3. Within the first $\sim 1.5 \mathrm{ML}$, the inverse lattice spacing remains virtually constant, although a small amount of relaxation may occur between 1 and 1.5 ML. Thus, the films are pseudomophic in this thickness range. Then the inverse lattice spacing starts to increase quickly, which is evidence of structural relaxation of the Fe films. This is consistent with the 3D growth that starts in the same thickness range as shown in Fig. 2. This inverse spacing approaches saturation at $\sim 3 \mathrm{ML}$ with a total relaxation of $5 \%-6 \%$. It is interesting to note that such relaxation increases the inverse spacing and therefore decreases the lattice spacing in real space. If we assume the epitaxy is for bcc $\mathrm{Fe}(110)$ to match the fcc $\operatorname{Pd}(110)$, then pseudomorphic bcc Fe should experience an in-plane strain of $\sim 4.2 \%$. Conventional wisdom, therefore, would suggest a relaxation toward the larger bulk Fe lattice constant and smaller inverse spacing, which disagrees with our experimental data. Another possibly is that the initial growth is fcc $\mathrm{Fe}$, whose lattice constant (extrapolated to a room temperature value of 3.59 $\AA$ ) is $8 \%$ smaller than that of the Pd (3.89 $\AA)$. So a fcc Fe phase at the initial growth would be consistent with the observed lattice relaxation, although it would represent a much larger lattice mismatch than for bcc Fe. A study of Fe growth on $\operatorname{Pd}(001)$ suggests that above $10 \mathrm{ML}$ the epitaxial Fe films are body-centered tetragonal; but the researchers could not fit their LEED current-voltage $(I / V)$ results at lower coverage. ${ }^{9}$ Our measurements cannot conclusively determine if the initial growth is bcc or fcc Fe.

Figure 4(a) shows a typical set of RHEED oscillations taken at: (i) the $(1,0)$ streak and at (ii) the center, and (iii) the tail of the $(0,0)$ streak as marked on Fig. 2. Both (i) and (ii) show the normal oscillation with 1 ML period in the first 1-2 ML. Similar oscillations are also observed on the $(2,0)$ streak. They indicate initial smooth, layer-by-layer growth of 
(a)

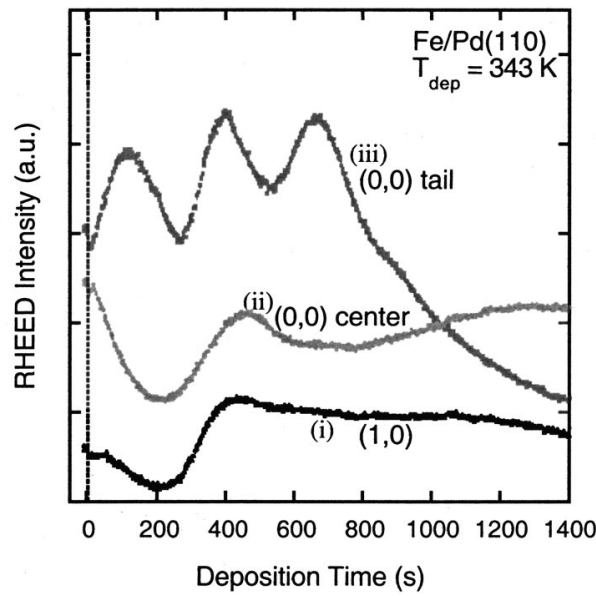

(b)

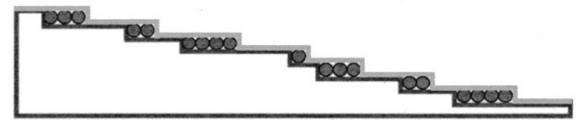

FIG. 4. (a) RHEED oscillations observed at: (i) the $(1,0)$ streak; (ii) the center of the $(0,0)$ streak; (iii) the low angle tail of the $(0,0)$ streak. (b) Schematic of the proposed growth mode for Fe on stepped Pd(110) with step decoration. Note that after $0.5 \mathrm{ML}$ Fe deposition, the terrace widths become significantly more uniform.

Fe on Pd(110). But (iii), taken at the very glancing angle, exhibits an oscillation with a $\frac{1}{2}$ ML period up to $1.5 \mathrm{ML}$. Such behavior can only be observed on the $(0,0)$ streak at the glancing exit angle end closest to the direct beam. It is well known that the phase and intensity of RHEED oscillation should vary at different angles or on different streaks. A change in periodicity is, however, difficult to explain by an ordinary layer-by-layer growth mode.

A peak at $0.5 \mathrm{ML}$ was first observed with atom beam scattering by Kern's group on a stepped surface. ${ }^{3,4}$ Suppose the starting substrate surface is stepped with a relatively broad terrace width distribution as illustrated in Fig. 4(b). If the adatoms are confined to their landing terrace and diffuse to the step edges to form smooth stripes, then at each given coverage, more atomic rows will appear on wider terraces than on the narrow ones. At around 0.5 ML, all the terraces would be half filled, giving rise to a much narrower distribution of terrace width as indicated in Fig. 4(b). When intensity oscillations of atom or electron beams are monitored with glancing incidence and exit angles, lateral structure is probed. A peak at $0.5 \mathrm{ML}$ therefore appears in the oscillation due to the change in lateral morphology. The fact that we observe $\frac{1}{2}$ ML oscillation suggests that step decoration occurs for this system. The width and spacing of the Fe stripes, however, are likely to have a relatively broad distribution due to irregularities in the substrate.

This system is ferromagnetic down to submonolayer Fe coverage. Figure 5 demonstrates the remanent magnetization measured with polar and longitudinal SMOKE at $42 \mathrm{~K}$. In both sets of measurements, remanent and saturation magnetization remain virtually the same (not shown). Polar Kerr signals are observed for as low as $\sim 0.3 \mathrm{ML}$ and persist up to $\sim 1.3 \mathrm{ML}$. They are strongly coverage dependent and peak at $\sim 0.6$ ML. Normally, for submonolayer films consisting of

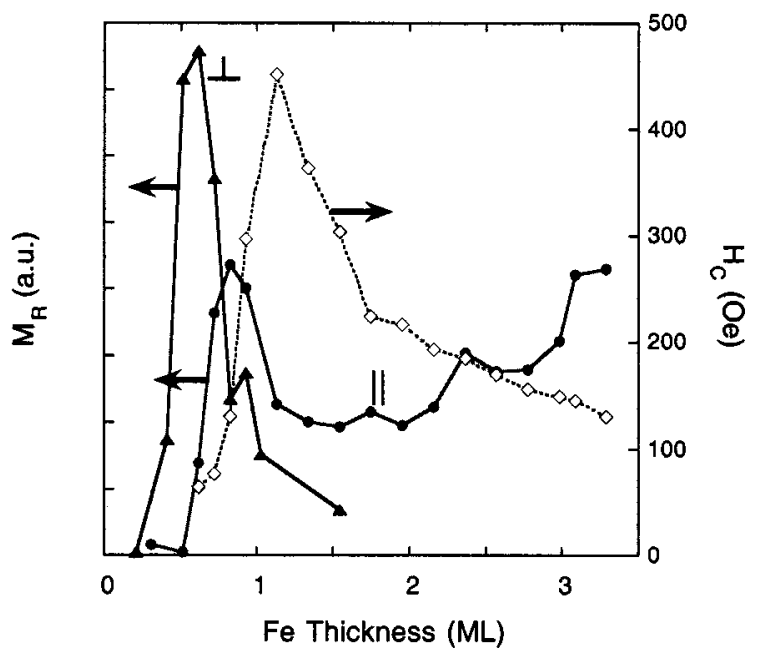

FIG. 5. Thickness dependence of the polar and longitudinal remanent magnetization along with the coercivity in the longitudinal signals measured with SMOKE.

small islands, ferromagnetic order starts to appear at island percolation coverage of $\sim 0.6 \mathrm{ML}$. One possibility for a lower ferromagnetic onset in our system is that the nearby $\mathrm{Pd}$ atoms could be polarized to make the magnetic islands larger and therefore coalesce earlier. Such an onset for $\mathrm{Fe} / \mathrm{Pd}(001)$, however, was observed between 0.5 and $1.0 \mathrm{ML}$ at low temperature, which corresponds to its island percolation seen with scanning tunneling microscopy. ${ }^{10}$ This demonstrates that Pd polarization does not contribute to an earlier onset, which is possibly because magnetic proximity is very short range and may only polarize the nearest $\mathrm{Pd}$ atoms directly below Fe atoms. Comparing Fe on $\mathrm{Pd}(110)$ and $\mathrm{Pd}(001)$, we suggest that the low ferromagnetic onset for $\mathrm{Fe} / \mathrm{Pd}(110)$ is related to the chain-like growth as discussed earlier.

Longitudinal signals, taken with the magnetic field along the step edges, are observed $\geqslant 0.5 \mathrm{ML}$. Above $\sim 1.5-2 \mathrm{ML}$, the longitudinal Kerr increases linearly with film thickness as expected. This region coincides with the start of 3D growth and lattice relaxation. Between 0.5 and $1.3 \mathrm{ML}$, an anomalous peak exists, where the Kerr signals are even larger than those of the thicker films. Its origin will be discussed in detail with additional experiments in the future. The results indicate that the magnetic easy axis at very low coverage of $0.3-0.5 \mathrm{ML}$ is perpendicular to the surface, while it is clearly in-plane above $1.5 \mathrm{ML}$. A theoretical calculation ${ }^{11}$ suggests that the easy axis of a freestanding Co chain is in-line to the chain, while it switches to perpendicular to the surface for $\mathrm{Co} / \mathrm{Pd}(110)$. No calculation, however, is available for $\mathrm{Fe} /$ $\operatorname{Pd}(110)$. At $\sim 0.9-1.5 \mathrm{ML}$, the coercive field of the in-plane magnetization significantly increases, an indication of mixed magnetic phases at a phase transition boundary. ${ }^{12}$

\section{CONCLUSIONS}

We have investigated $\mathrm{Fe}$ growth on a stepped $\mathrm{Pd}(110)$ surface and the magnetic properties at 0-3 ML. RHEED oscillations suggest that step decoration occurs in the first 1.5 ML, when the films are pseudomorphic. The Fe films are 
ferromagnetic even at low submonolayer coverage, and initially have a magnetic easy axis perpendicular to the surface. Since such a system seems to be quasi-1D, it will be interesting to further investigate the submonolayer magnetic properties in the future.

\section{ACKNOWLEDGMENTS}

This work was supported by DOE BES-MS Grant No. W-31-109-ENG-38. The authors thank W. Keune for valuable discussions. One of us, B.R.C., would like to thank the Department of Physics of the Gerhard-Mercator-Universität Duisburg for travel support.

${ }^{1}$ See, for example, articles in J. Magn. Magn. Mater. 200 (1999).

${ }^{2}$ F. J. Himpsel, T. Jung, A. Kirakosian, J.-L. Lin, D. Y. Petrovykh, H. Rauscher, and J. Viernow, MRS Bull. 24, 20 (1999); F. J. Himpsel, T. Jung, and J. E. Ortega, Surf. Rev. Lett. 4, 371 (1997).
${ }^{3}$ P. Gambardella, M. Blanc, H. Brune, K. Kuhnke, and K. Kern, Phys. Rev. B 61, 2254 (2000).

${ }^{4}$ P. Gambardella, M. Blanc, L. Bürgi, K. Kuhnke, and K. Kern, Surf. Sci. 449, 93 (2000).

${ }^{5}$ H. J. Elmers, J. Hauschild, H. Höche, U. Gradmann, H. Bethge, D. Heuer, and U. Köhler, Phys. Rev. Lett. 73, 898 (1994); J. Hauschild, H. J. Elmers, and U. Gradmann, Phys. Rev. B 57, R677 (1998).

${ }^{6}$ J. Shen, M. Klaua, P. Ohresser, H. Jrnniches, J. Barthel, C. V. Mohan, and J. Kirschner, Phys. Rev. B 56, 11134 (1997).

${ }^{7}$ J.-P. Bucher, E. Hahn, P. Fernandez, C. Massobrio, and K. Kern, Europhys. Lett. 27, 473 (1994).

${ }^{8}$ H. J. Choi, R. K. Kawakami, E. J. Escorcia-Aparicio, Z. Q. Qiu, J. Pearson, J. S. Jiang, Dongqi Li, and S. D. Bader, Phys. Rev. Lett. 82, 1947 (1999), references therein.

${ }^{9}$ J. Quinn, Y. S. Li, H. Li, D. Tian, F. Jona, and P. M. Marcus, Phys. Rev. B 43, 3959 (1991).

${ }^{10}$ X. F. Jin, J. Barthel, J. Shen, S. S. Manoharan, and J. Kirschner, Phys. Rev. B 60, 11809 (1999).

${ }^{11}$ J. Dorantes-Dávila and G. M. Pastor, Phys. Rev. Lett. 81, 208 (1998).

${ }^{12}$ Dongqi Li, M. Freitag, J. Pearson, Z. Q. Qiu, and S. D. Bader, Phys. Rev. Lett. 72, 3112 (1994); S. D. Bader, Dongqi Li, and Z. Q. Qiu, J. Appl. Phys. 76, 6419 (1994). 